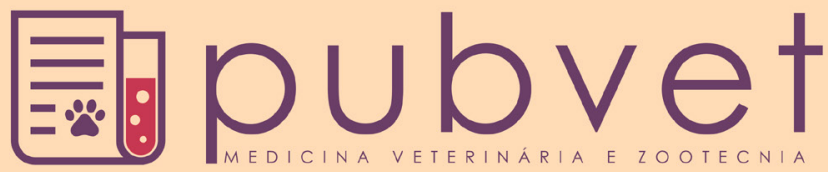

HTTP://DX.DOI.ORG/10.22256/PUBVET.V11N6.616-619

\section{Parâmetros ultrassonográficos e clínicos em caso de lipidose hepática felina: Relato de caso}

\author{
Érika Souza Vieira ${ }^{1}$, Otávia Augusta de Mello², Matheus Batista de Oliveira $^{3 *}$ \\ 'Professora da Faculdade Pio Décimo, Sergipe, Brasil. E-mail: kinhasouza@gmail.com \\ ${ }^{2}$ Graduanda em Medicina Veterinária pela Faculdade Pio Décimo, Sergipe, Brasil: E-mail: otaviamello1@gmail.com \\ ${ }^{3 *}$ Graduando em Medicina Veterinária pela Faculdade Pio Décimo, Sergipe, Brasil.E-mail: matheuslateral@hotmail.com \\ *Autor para a correspondência
}

\begin{abstract}
RESUMO. A lipidose hepática felina é uma das hepatopatias mais comuns e potencialmente fatal que acomete gatos domésticos. Na maioria das vezes afeta gatos privados de alimento ou que passaram por períodos prolongados de anorexia. É um processo patológico que atinge gatos adultos, podendo estar associado a colangiohepatite, obstruções biliares ou neoplasia intra-hepática ou apresentar caráter idiopático. Normalmente, os ácidos graxos que circulam no fígado são captados e metabolizados para produzir energia ou se convertem em triglicérides e são segregados de novo para a circulação. A doença pode se apresentar como resultado de uma desordem na oxidação dos ácidos graxos pelos hepatócitos, ou da incapacidade do fígado para segregar as lipoproteínas encarregadas de transportar as triglicérides através da corrente sanguínea. A doença é bastante comum, e tende a afetar gatos obesos. A anorexia prolongada pode causar acúmulo significativo de gordura no fígado e o aparecimento das manifestações clínicas. Qualquer que seja a causa metabólica da lipidose hepática, a recuperação do animal requer diagnóstico precoce, início imediato da terapia sintomática e sonda de alimentação para suporte nutricional.
\end{abstract}

Palavras chaves: Anorexia, hepatócitos, triglicérides, obesidade

\section{Ultrasonographic and clinical parameters in the case of feline hepatic lipidosis: Case report}

ABSTRACT: Feline hepatic lipidosis is one of the most common and potentially fatal hepatopathies that affects domestic cats. Most of the time it affects cats deprived of food or who have gone through prolonged periods of anorexia. It is a pathological process that affects adult cats, and may be associated with cholangiohepatitis, biliary obstruction or intrahepatic neoplasia or idiopathic character. Normally, the fatty acids that circulate in the liver are picked up and metabolized to produce energy or converted to triglycerides and are segregated back into the circulation. The disease may occur as a result of a disorder in the fatty acid oxidation by hepatocytes, or the inability of the liver to secrete the lipoproteins in charge of transporting the triglycerides through the bloodstream. The disease is quite common, and tends to affect obese cats. Prolonged anorexia can cause significant accumulation of fat in the liver and the onset of clinical manifestations. Whatever the metabolic cause of hepatic lipidosis, recovery of the animal requires early diagnosis, immediate initiation of symptomatic therapy, and feeding probe for nutritional support.

Keywords: Anorexia, hepatocytes, triglycerides, obesity 


\section{Parámetros ultrasonográficos y clínicos en caso de liposis hepática felina: Reporte de un caso}

RESUMEN. La liposis hepática felina es una de las hepatopatías más comunes y potencialmente fatal que acomete gatos domésticos. La mayoría de las veces afecta a gatos privados de alimentos o que han pasado por períodos prolongados de anorexia. Es un proceso patológico que afecta a gatos adultos, pudiendo estar asociado a colangiohepatitis, obstrucciones biliares o neoplasia intrahepática o presentar un carácter idiopático. Normalmente, los ácidos grasos que circulan en el hígado son captados y metabolizados para producir energía o se convierten en triglicéridos y se segregan de nuevo para la circulación. La enfermedad puede presentarse como resultado de un desorden en la oxidación de los ácidos grasos por los hepatocitos, o de la incapacidad del hígado para segregar las lipoproteínas encargadas de transportar los triglicéridos a través del torrente sanguíneo. La enfermedad es bastante común, y tiende a afectar a los gatos obesos. La anorexia prolongada puede causar una acumulación significativa de grasa en el hígado y la aparición de las manifestaciones clínicas. Cualquiera que sea la causa metabólica de la liposis hepática, la recuperación del animal requiere diagnóstico precoz, inicio inmediato de la terapia sintomática y sonda de alimentación para soporte nutricional.

Palabras claves: Anorexia, hepatocitos, triglicéridos, obesidad

\section{Introdução}

A Lipidose Hepática Felina (FHL) foi descrita pela primeira vez por (Barsanti et al., 1977) como uma afecção idiopática. Sabe-se que a maioria dos gatos afetados (mais de 95\%) sofre de um processo patológico primário ou secundário e que desencadeiam um estado catabólico, dentre elas podemos citar as colangite/colangiohepatite, oclusão extra-hepáticas do ducto biliar, neoplasias (Center, 2005). Segundo Nicoll et al. (1998) ocorre frequentemente em gatos obesos e que sofreram um período de anorexia ou uma privação alimentar prolongada.

O estilo de vida sedentário, o confinamento a ambiente indoor e a esterilização, são fatores que contribuem para o desenvolvimento da obesidade, pois há diminuição da atividade física bem como alteração das taxas metabólicas, as quais exacerbam o desequilíbrio entre a energia ingerida e a energia consumida. A maioria dos gatos afetados é adulta, com mais de dois anos e não há predisposição sexual ou racial. Os gatos afetados são comumente obesos e sofreram há pouco um evento estressante, em decorrência do qual se tornaram anoréticos e perderam peso com rapidez. Raramente se identifica o evento desencadeante (August, 1993). Em possa afetar gatos de qualquer idade, a doença surge mais comumente entre os quatro e os quinze anos. Existem vários mecanismos fisiopatológicos que estão na base do desenvolvimento da FHL: o aumento do fluxo de ácidos graxos livres para o fígado, o aumento da síntese hepática de ácidos graxos, as alterações na oxidação dos ácidos graxos no hepatócito e a diminuição do transporte pelas lipoproteínas de muito baixa densidade (Szabo et al., 2000).

Brown et al. (2000) propõem como causas o aumento da lipólise periférica secundária a uma deficiência absoluta ou relativa em insulina, o fígado gordo induzido por má nutrição proteicocalórica (similar ao kwashiorkor ou marasmus em humanos), a deficiência em aminoácidos essenciais (arginina e metionina) a que dá origem a uma incapacidade de sintetizar apoproteínas suficientes para mobilizar os lipídios hepáticos, a deficiência em compostos lipotróficos, a deficiência congénita ou adquirida da oxidação dos ácidos gordos e a lesão peroxissomal hepática devido a stress oxidativo.

\section{Relato de caso}

Em 19/09/2016, um felino SRD, de cinco anos de idade, pesando $8 \mathrm{~kg}$, chegou ao Hospital Veterinário Dr. Vicente Borelli da Faculdade Pio Décimo, em Aracaju - SE. O tutor mencionou que o animal estava apático e com perda de apetite. Ao exame clínico apresentou sinais de doença do trato urinário inferior felino (DTUIF) e que, após três dias, retornou ao hospital apresentando estenose, sendo indicado a uretrostomia. Durante o período pós-cirúrgico, o animal apresentou anorexia e êmese, com perda de $2 \mathrm{~kg}$ de seu peso inicial. Foram solicitados exames complementares bioquímicos de perfil hepático e renal e exame ultrassonográfico de cavidade abdominal, com objetivo de visualizar, particularmente, o fígado. 


\section{Resultados e Discussão}

O fígado possui funções diversas, relacionadas ao fluxo sanguíneo hepático; o metabolismo de proteínas, carboidratos e gorduras; desintoxicação e excreção de drogas e toxinas (Birchard and Sherding, 2008). A Lipidose Hepática Felina LHF é uma condição clínica decorrente de uma série de fatores, dentre eles, o excesso de gordura localizada em região hepática, denominada esteatose.

O exame ultrassonográfico foi fundamental, pois se verificou a presença de gordura disseminada por todo o órgão (hiperecogenicidade), sendo diagnosticado como esteatose hepática em grau III e hepatomegalia (Figura 1). Os rins revelaram topografia habitual e

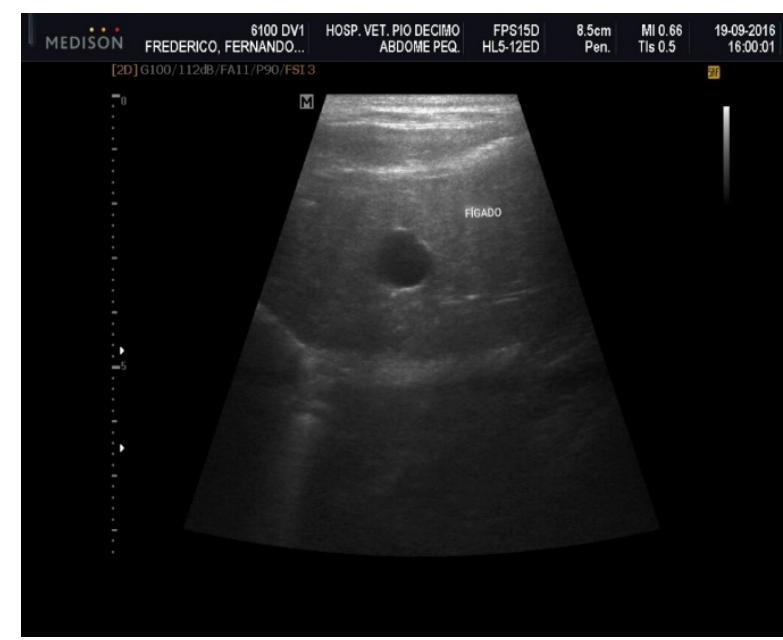

Figura 1. Imagem ul trassonográfica do figado do felino, 5 anos com suspeita clinica de lipidose. Aumento do tamanho do órgão, parênquima homogêneo, hiperecogênico, caracteristico de hepatomegalia e estea tose. contornos regulares, entretanto, a relação córticomedular se apresentava pouco definida, confirmando uma nefropatia (Figura 2).

A correlação do exame bioquímico renal, o qual se mostrou alterado (Tabela 1), e o exame ultrassonográfico, confirmando nefropatia, estão relacionados diretamente ao quadro em que se encontrava o felino, uma vez que clinicamente foi confirmado doença do trato urinário inferior felino (DTUIF). Porém, a principal patologia era no fígado, que resultou em lipidose metabólica. No entanto, a patologia do sistema urinário que o felino possuía, foi uma das causas que desencadeou a perda de apetite e, consequente, metabolização de lipídios pelo organismo, lipidose.

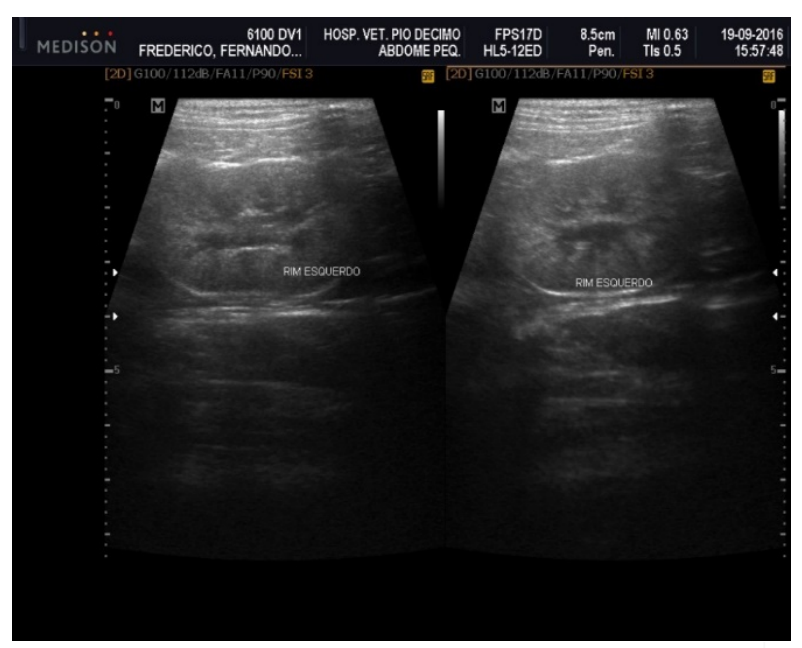

Figura 2. Imagem ultrassonográfica dos rins do felino, 5 anos com suspeita clinica de lipidose, relação córticomedular pouco de finida, ne fropatia.

Tabela 1. Exame bioquímico do perfil hepático e renal do felino, 5 anos com suspeita clínica de lipidose

\begin{tabular}{lcc}
\hline Perfil hepático/ renal & Resultado (UI/L) & Referência \\
\hline ALT/TGP & 14 & $(10-88)$ \\
AST/TGO & 15 & $(10-88)$ \\
Ureia & $47(\uparrow)$ & $(10-20)$ \\
Creatinina & 1,7 & $(0.8-1,8)$ \\
\hline
\end{tabular}

\section{Conclusão}

A opção do caso clínico de lipidose hepática felina foi relevante por referir-se à uma patologia sempre presente na rotina clínica veterinária de pequenos animais, a qual exige muito conhecimento do clínico a respeito da problemática, através de mecanismos e formas para concluir o diagnóstico. Por isso, faz-se importante o uso da ultrassonografia em âmbito veterinário, destacando-se pela sua importante contribuição, junto a outros tipos exames, no diagnóstico da lipidose hepática felina.

Deste modo, alerta-se para o aumento do número de casos de lipidose em felinos, onde maioria não são diagnosticados precisamente, por falta de conhecimento do clínico a respeito das 
ferramentas de diagnóstico complementar, tanto o bioquímico, assim como, e mais relevante, o exame ultrassonográfico.

\section{Referências Bibliográficas}

August, J. R. 1993. Enfermedades del oído. Las Clínicas Vetrinárias de Norteamerica, 18, 1274.

Barsanti, J. A., Jones, B. D., Spano, J. S. \& Taylor, H. W. 1977. Prolonged anorexia associated with hepatic lipidosis in 3 cats. Feline Practice, 7, 52-57.

Birchard, S. J. \& Sherding, R. G. 2008. Manual Saunders: clínica de pequenos animais, São Paulo.

Brown, B., Mauldin, G. E., Armstrong, J., Moroff, S. D. \& Mauldin, G. N. 2000. Metabolic and hormonal alterations in cats with hepatic lipidosis. Journal of Veterinary Internal Medicine, 14, 20-26.

Center, S. A. 2005. Feline hepatic lipidosis. Veterinary Clinics of North America: Small Animal Practice, 35, 225-269.
Nicoll, R. G., Jackson, M. W., Knipp, B. S., Zagzebski, J. A., Steinberg, H. \& O'Brien, R. T. 1998. Quantitative ultrasonography of the liver in cats during obesity induction and dietary restriction. Research in Veterinary Science, 64, 1-6.

Szabo, J., Ibrahim, W. H., Sunvold, G. D., Dickey, K. M., Rodgers, J. B., Toth, I. E., Boissonneault, G. A. \& Bruckner, G. G. 2000. Influence of dietary protein and lipid on weight loss in obese ovariohysterectomized cats. American Journal of Veterinary Research, 61, 559-565.

\section{Article History:}

Received 21 February 2017

Accepted 27 March 2017

Available on line 31 May 2017

License information: This is an open-access article distributed under the terms of the Creative Commons Attribution License 4.0, which permits unrestricted use, distribution, and reproduction in any medium, provided the original work is properly cited. 